\title{
Predictive factors of disease-free survival after complete pathological response to neoadjuvant radiotherapy for rectal adenocarcinoma: retrospective case series
}

Amine Souadka ${ }^{1 *}$ (D), Mohammed Anass Majbar ${ }^{1}$, Amine Benkabbou' ${ }^{1}$, Badr Serji ${ }^{2}$, Tarik Souiki ${ }^{3}$, Sidi Mohammed Bouchentouf ${ }^{4}$, Mourad Abid ${ }^{5}$, Basma El Khannousi ${ }^{6}$, Tijani El Harroudi ${ }^{2}$, Hadj Omar El Malki ${ }^{7}$, Mohammed Raiss ${ }^{6}$, Lahsen Ifrine', Khalid Mazaz ${ }^{3}$, Aziz Zentar ${ }^{4}$, Raouf Mohsine ${ }^{1}$, Abdelilah Souadka ${ }^{8}$, Abdelkader Belkouchi ${ }^{7}$, Mohammed Ahallat, Abdelmalek Hrora ${ }^{1,9}$ and on behalf of the Moroccan Society of Surgery

\begin{abstract}
Background: Many data suggest that patients with low rectal adenocarcinoma who achieved ypTONO status have improved survival and disease-free survival (DFS) compared to all other stages however only few data are available regarding the specific prognosis factors of this subgroup. This study aimed to evaluate predictive factors for disease free survival after complete pathological response (CPR) in cases of low rectal adenocarcinoma.

Materials and methods: From January 2005 to December 2013, all patients with low rectal adenocarcinoma who underwent neoadjuvant chemoradiotherapy followed by total mesorectal excision and achieved CPR were included at 7 Moroccan and 1 Algerian centres. Predictive factors for disease-free survival were analysed by uni and multivariate analysis.

Results: Eigthy-four (12.1\%) patients achieved a CPR (ypTONO). Multivariate analysis revealed that both poorly differentiated tumors $(\mathrm{OR}, 9.23 ; 95 \mathrm{Cl} 1.35-62.82 ; P=0.023)$ and the occurrence of perineal sepsis $(\mathrm{OR}, 13.51 ; 95 \mathrm{Cl}$ $1.96-93.12 ; P=0.008$ ) were independently associated with impaired DFS.

Conclusions: Patients with low rectal cancer who exhibited a CPR after neoadjuvant therapy have good prognoses; however, the occurrence of perineal sepsis and/or poor initial differentiation may be associated with impaired DFS in these patients.

Trial registration: The study was retrospectively registered the 28th July 2018 in ClinicalTrials.gov register with the reference NCT03601689.
\end{abstract}

Keywords: Rectal neoplasm, Neoadjuvant treatment, Complete pathological response, Disease-free survival, Predictive factors

\footnotetext{
* Correspondence: a.souadka@um5s.net.ma

${ }^{1}$ Surgical Oncology Department, National Institute of Oncology, Mohammed

$\checkmark$ University Medical School, Rabat, Morocco

Full list of author information is available at the end of the article
}

(c) The Author(s). 2019 Open Access This article is distributed under the terms of the Creative Commons Attribution 4.0 International License (http://creativecommons.org/licenses/by/4.0/), which permits unrestricted use, distribution, and reproduction in any medium, provided you give appropriate credit to the original author(s) and the source, provide a link to the Creative Commons license, and indicate if changes were made. The Creative Commons Public Domain Dedication waiver (http://creativecommons.org/publicdomain/zero/1.0/) applies to the data made available in this article, unless otherwise stated. 


\section{Background}

Currently, the standard treatment for mid-low locally advanced rectal cancer is neoadjuvant (chemo)-radiation therapy followed by curative surgical resection according to the guidelines of both the European Society of Medical Oncology (ESMO) and the National Comprehensive Cancer Network (NCCN) [1]. Most patients exhibit a substantial downstaging that may lead to complete pathological responses (CPRs) in 15 to $20 \%$ of cases, as defined by the absence of viable tumour cells after full pathologic examination of the resected specimen (ypT0N0), which is referred to as stage 0 disease [2]. These findings have helped many authors to reconsider the need for surgical resection after a CPR [3-6]; however, no definitive surrogate of a CPR (clinical, biological or radiological) has been reported in the literature, and surgical resection remains the standard treatment.

Although many data suggest that patients with ypT0N0 status have better prognoses and improved survival and disease-free survival (DFS) compared to all other stages, few data are available regarding the details of oncological outcomes [7, 8]. Additionally, little is known about the specific prognostic factors for this subgroup of patients.

The aim of this study was to evaluate the long-term oncologic outcomes and predictive factors for DFS after a CPR of low rectal adenocarcinoma.

\section{Methods}

\section{Study design}

This was a retrospective multicenter case series study conducted by the Moroccan Society of Surgery. Eight centres agreed to participate: seven were in Morocco (i.e., the Surgical Departments A and C, Ibn Sina Hospital, the National Institute of Oncology, the Military Hospital in Rabat, the Surgical Department B in Hassan 2 University Hospital in Fes, the Oncological Surgical Department in Oujda and a private oncological centre), and one was located in Algeria (the Anticancer Centre, Batna).

An online form (Google forms) was sent to each participating centre for data collection, and all information was anonymous. Each investigator obtained ethical approval from their own centre. This study was reviewed and approved by the Ethics Committee of the Ibn Sina Hospital (Rabat, Morocco). The study was registered in ClinicalTrials.gov register with the reference NCT03601689 and has been reported in line with the PROCESS criteria [9].

\section{Inclusion and exclusion criteria}

The inclusion criteria were as follows: (1) patients over 18 years of age with a histologically proven low rectal adenocarcinoma, (2) no previous or synchronous colorectal disease, (3) UICC stage I-III patients who underwent neoadjuvant chemoradiotherapy, (4) chemotherapy followed by total mesorectal excision (TME), and (5) a CPR defined as ypTONO.

The exclusion criteria were as follows: patients with metastatic tumour's or missing data.

\section{Endpoints}

This study primarily aimed to analyse the local and distant recurrence rates and secondarily aimed to determine the predictive factors of DFS.

\section{Therapeutic protocol}

All patients from Moroccan centres underwent preoperative radiotherapy $(50.4 \mathrm{~Gy})$ and concomitant chemotherapy (Capecitabine $825 \mathrm{mg} / \mathrm{mg}$ twice daily). Patients in the Algerian centre were enrolled in a prospective study with a short protocol of $5 \times 5$ Gy radiotherapy. All patients underwent surgery at least $6-8$ weeks after the end of neoadjuvant treatment.

Tumours up to 3 to $4 \mathrm{~cm}$ underwent an anterior resection with stapled colorectal or manual coloanal anastomosis, whereas smaller tumours with no invasion of the external sphincter underwent an intersphincteric resection. In cases with no distal margin or external sphincter involvement, an abdominoperineal resection (APR) was performed, followed by either an iliac colostomy or perineal pseudocontinent colostomy [10].

Because all selected patients achieved a CPR, no adjuvant treatments were administered, according to the guidelines.

\section{Pathological assessment}

A CPR was defined as a pathological report of the surgical specimens describing a status of ypT0N0 according to the Dworak classification [11]. All other cases (ypN+ and/or ypT+) were considered non-responses and were excluded.

The specimens were analysed using very similar protocols in each institute (i.e., 5-mm slices of the rectal tumours were subjected to intensified evaluations of the tissue at the tumour site and at 2 to 3 sublevels in cases in which no tumour was found in the initial block). A second pathologist reviewed all CPR surgical specimens.

\section{Early postoperative outcomes}

The early postoperative outcomes included the inhospital and/or one-month postoperative periods. Complications were evaluated according to the ClavienDindo classification [11].

Perineal sepsis was defined as the presence of a postoperative clinical anastomotic fistula (pus or faecal 
discharge from the drain, pelvic abscess, peritonitis, recto-vaginal fistula, or discharge of pus from the rectum) in cases of colorectal or coloanal anastomoses and as perineal infection (i.e., the presence of a pelvic abscess or wound dehiscence) in cases of APR. [12]

\section{Assessment of oncologic outcomes}

Patients were followed up alternately by a surgeon and an oncologist via a clinical examination, a stoma examination and a liver ultrasound or thoracoabdominopelvic CT examination every three to 4 months for 2 years, every 6 months for 3 years after that, and once per year thereafter. A postoperative recurrence was defined by biopsy-proven or radiographic evidence of local or distant recurrent disease. DFS was defined as the period between the day of surgery and the date of recurrence or the last date of follow-up.

\section{Statistical analysis}

Continuous variables are presented as the means \pm SDs or as the medians with the interquartile ranges, and categorical variables are expressed as frequencies and percentages. SPSS software (SPSS 13.0; SPSS Inc., Chicago, IL) was used for the univariate and multivariate analyses that were applied to identify the predictive factors for recurrence in patients with ypTONO status.

Only patients with sufficient follow-up were included in the analysis of the predictive factors of DFS (patients who died postoperatively and those lost to follow up were excluded from this analysis). The analysed variables were age, sex, ASA score, distance from the anal verge, differentiation degree at the initial rectal biopsy, $\mathrm{T}$ and $\mathrm{N}$ pre-therapeutic stages, the type of neoadjuvant radiotherapy, the median interval between preoperative CRT completion and surgery, the type of surgical procedure and the occurrence of perineal sepsis. Comparisons between groups were performed using the $\mathrm{X}^{2}$ test or Fisher's exact test as appropriate. All variables associated with a poor functional result with a $P$ value equal to or less than 0.1 in the univariate analysis were introduced into a multivariate logistic regression model that included the calculations of the ORs and 95\% CIs. A P value of $<0.05$ was considered statistically significant.

Survival was analysed according to the Kaplan-Meier method. The predictive factors of DFS were analysed by Cox regression.

\section{Results}

From January 2005 to December 2013, 694 consecutive patients underwent neoadjuvant treatment followed by TME in the 8 centres.

Of these, 84 (12.1\%) patients achieved a CPR (ypT0N0). The mean age of these patients was 54.5 years
(SD 12 years). The demographic details and treatment modalities are provided in Tables 1 and 2 .

The 30-day mortality rate was $3.6 \%$, and the global complication rate as defined by a Clavien-Dindo score (CD) $\geq$ IIIa was $14.3 \%$. Perineal sepsis occurred in 16 patients (19\%).

Table 1 Demographics and surgical procedures in 84 patients with complete pathological response (CPR) after neoadjuvant treatment

\begin{tabular}{|c|c|}
\hline Characteristics & $\mathrm{N}(\%)$ \\
\hline \multicolumn{2}{|l|}{ Gender } \\
\hline Male & $38(45.2)$ \\
\hline female & $46(54.8)$ \\
\hline Mean age \pm SD (years) & $55,2 \pm 12,5$ \\
\hline \multicolumn{2}{|l|}{ ASA score } \\
\hline 1 & $48(57)$ \\
\hline 2 & $13(15.5)$ \\
\hline Missing & $23(27.5)$ \\
\hline Median distance from the anal verge (cm) (quartiles) & $4(3-6)$ \\
\hline \multicolumn{2}{|l|}{ Histologic differentiation } \\
\hline Well differentiated & $56(66.7)$ \\
\hline Poorly differentiated & $14(16.7)$ \\
\hline Missing & $14(16.7)$ \\
\hline \multicolumn{2}{|l|}{ Pretherapeutic T stage } \\
\hline $\mathrm{T} 1-\mathrm{T} 2$ & $19(22)$ \\
\hline T3- T4 & $52(62)$ \\
\hline missing & $13(15.5)$ \\
\hline \multicolumn{2}{|l|}{ Pretherapeutic N stage } \\
\hline NO & $20(23.8)$ \\
\hline N1 & $53(63.1)$ \\
\hline Missing & $11(13.1)$ \\
\hline \multicolumn{2}{|l|}{ Neoadjuvant radiotherapy } \\
\hline Concomitant chemotherapy & $76(90.5)$ \\
\hline 45Gy & $6(7.2)$ \\
\hline 25 Gy & $2(2.5)$ \\
\hline Median delay CRT/ Surgery (weeks) & $7(6-8)$ \\
\hline \multicolumn{2}{|l|}{ Surgical approach } \\
\hline Laparoscopy & $26(31)$ \\
\hline Open procedure & $58(69)$ \\
\hline \multicolumn{2}{|l|}{ Surgical procedures n(\%) } \\
\hline Anterior resection & $51(60.8)$ \\
\hline Coloanal anastomosis & $31(37)$ \\
\hline Colorectal anastomosis & $20(23.8)$ \\
\hline APR & $33(39.3)$ \\
\hline Left Iliac colostomy & $23(27.4)$ \\
\hline Perineal pseudocontinent colostomy & $10(11.9)$ \\
\hline
\end{tabular}


Table 2 Thirty-days surgical outcomes and pathological details in 84 patients with complete pathological response (CPR) after neoadjuvant treatment

\begin{tabular}{ll}
\hline Thirty days surgical outcomes & $\mathrm{N}(\%)$ \\
\hline Mortality & $3(3.6)$ \\
Global complications & $25(29.8)$ \\
$\quad<$ Illa & $13(15.5)$ \\
$\geq$ Illa & $12(14.3)$ \\
Perineal sepsis & $16(19)$ \\
Pathological details & \\
Median size of the scar cm (range) & $2(0-10)$ \\
Median Distal margins in cm (Range) & $2.35(0-8)$ \\
Median Circumferential margins in mm (range) & $2(0-20)$ \\
Median lymph node number $\mathrm{n}$ (range) & $8(0-22)$ \\
Acellular mucine $\mathrm{n}(\%)$ & $15(18)$ \\
\hline
\end{tabular}

\section{Pathological results}

The pathological examinations revealed that the median tumour scar was $2 \mathrm{~cm}$ with median distal and circumferential margins of $2.35 \mathrm{~cm}$ and $2 \mathrm{~mm}$, respectively. Fifteen patients (18\%) had residual acellular mucin, and the median number of retrieved lymph nodes was 8 (range $0-21$ ).

\section{Oncologic outcomes}

Two patients were lost to follow up, and 3 patients died postoperatively and were excluded from the oncologic analysis. The median follow-up duration was 30 months (range: 3 to 120 months).

Of the 79 patients, 9 patients developed recurrence (11.4\%), including 4 local recurrences, 3 distant recurrences and 2 simultaneous local and distant recurrences.

\section{Predictors of disease-free survival}

The univariate analysis indicated that poorly differentiated tumours (OR, 10.75;95 CI 1.90-58.67; $P=0.007$ ) and the occurrence of perineal sepsis (OR, 7.32; $95 \mathrm{CI}$ $1.81-29.50 ; P=0.005)$ were significantly associated with recurrences. The other variables that were evaluated (i.e., age, sex, ASA score, distance from the anal verge, $\mathrm{T}$ and $\mathrm{N}$ pre-therapeutic stages, type of neoadjuvant radiotherapy, the median delay to CRT surgery and the type of surgical procedure) were not associated with impaired DFS. (Table 3).

In the multivariate analysis, both poorly differentiated tumours (OR, 9.23; $95 \mathrm{CI} 1.35-62.82 ; P=0.023)$ and the occurrence of perineal sepsis (OR, 13.51; 95 CI 1.9693.12; $P=0.008)$ were independently associated with impaired DFS. (Figs. 1 and 2).

Table 3 Univariate and multivariate analysis of predictive factors of impaired disease-free survival

\begin{tabular}{|c|c|c|c|c|}
\hline & \multicolumn{2}{|l|}{ Univariate analysis } & \multicolumn{2}{|c|}{ Multivariate analysis } \\
\hline & $\mathrm{HR}(95 \% \mathrm{Cl})$ & $P$ & $\mathrm{HR}(95 \% \mathrm{Cl})$ & $P$ \\
\hline Gender & & 0.79 & & \\
\hline Male & 1 & & & \\
\hline Female & $1.2(0.32-4,45)$ & & & \\
\hline Histologic differentiation & & 0.007 & & 0.02 \\
\hline Well differentiated & 1 & & 1 & \\
\hline Poorly differentiated & $10.75(1.90-58.67)$ & & $9.23(1.36-62.82)$ & \\
\hline Pretherapeutic T stage & & 0.94 & & \\
\hline T1-T2 & 1 & & & \\
\hline T3-T4 & $0.94(0.17-5.17)$ & & & \\
\hline Pretherapeutic N stage & & 0.66 & & \\
\hline No & 1 & & & \\
\hline N1 & $0.68(0.12-3.72)$ & & & \\
\hline Neoadjuvant treatment & & 0.66 & & \\
\hline radiotherapy alone & 1 & & & \\
\hline Chemoradiotherapy & $0.61(0.07-5.43)$ & & & \\
\hline Surgical procedure & & 0.89 & & \\
\hline APR & 1 & & & \\
\hline Conservative intervention & $1.1(0.29-4.10)$ & & & \\
\hline Perineal sepsis & & 0.005 & & 0.008 \\
\hline No & 1 & & 1 & \\
\hline Yes & $7.32(1.81-29.50)$ & & $13.51(1.96-93.12)$ & \\
\hline
\end{tabular}




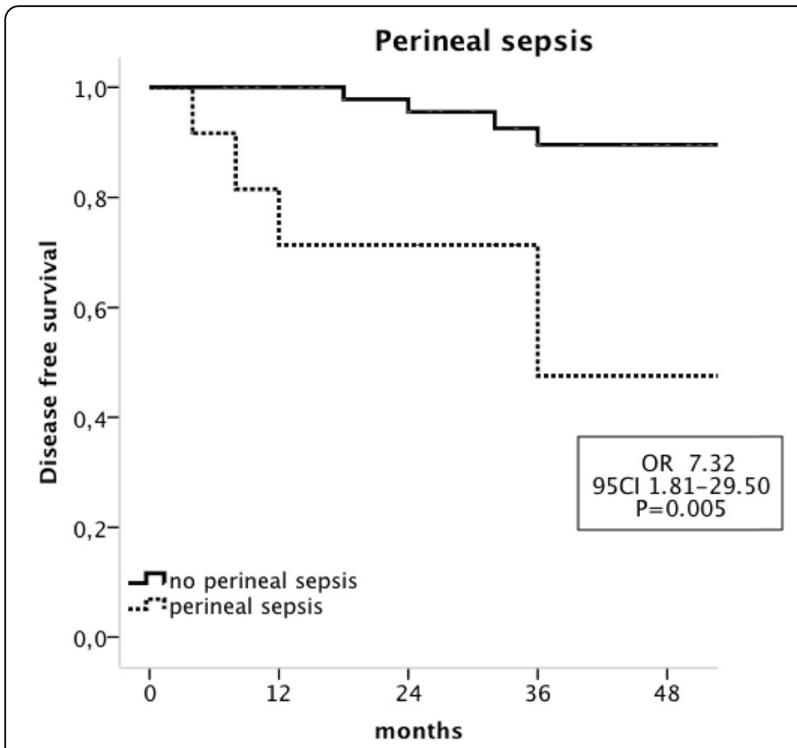

Fig. 1 Kaplan-Meier analysis of Disease free survival in patients with CPR according to the occurrence of perineal sepsis

\section{Discussion}

This study demonstrated that for patients with a CPR after neoadjuvant treatment followed by TME, the recurrence rate was $12.6 \%$. Both poorly differentiated tumours and the occurrence of perineal sepsis were factors that independently predicted impaired DFS in this population with ORs of 9.23 and 13.51, respectively.

Patients who develop a CPR after neoadjuvant treatment exhibit better prognoses, a reduced propensity for local or distant recurrence and improved survival $[4,13$, 14]. However, one study reported that even for

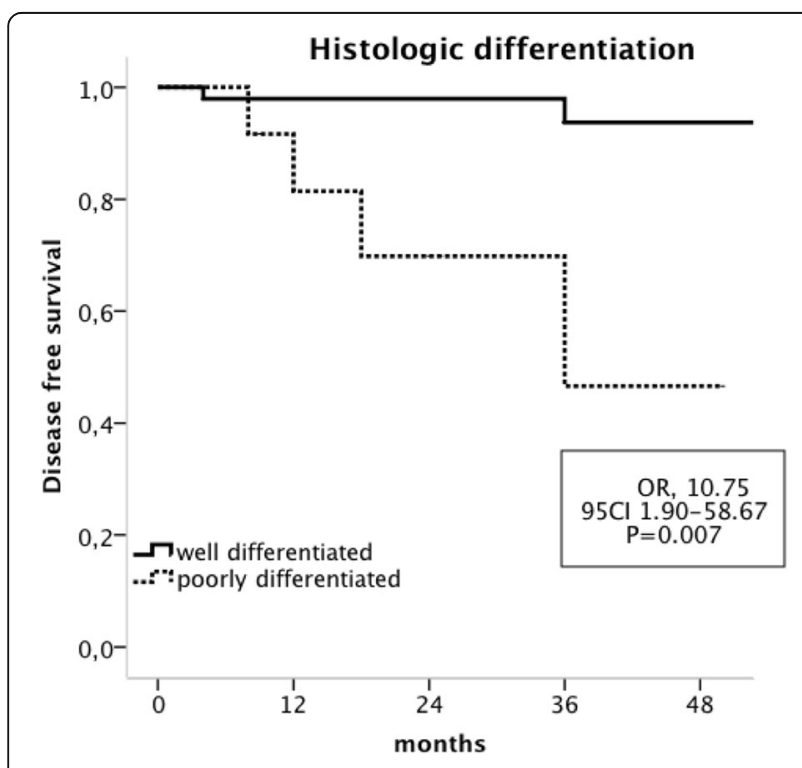

Fig. 2 Kaplan-Meier analysis of Disease free survival in patients with CPR according to the pretherapeutic histologic differentiation
ypTONOM0 low rectal cancers, after a median follow-up of 48 months, 61 of 419 patients developed recurrences, which resulted in a five-year DFS of $83 \%$ [4].

A CPR after chemoradiation therapy reduces, but does not eradicate, the risks of local and distant metastases $[4,6,7]$. Additionally, there are no data regarding the predictive factors of impaired DFS in this specific population of patients with good prognoses who achieved a CPR for low rectal adenocarcinoma.

Growing evidence and accumulating data indicate that anastomotic leakage (perineal sepsis) affects not only the short- but also the long-term survival [15]. In a recent meta-analysis, anastomotic leakage was associated with a greater rate of local recurrence (HR 1.71; 95\% CI 1.222.38 ) and a decrease in overall survival (HR 1.67; 95\% CI 1.19-2.35) [16]. A major explanation of this finding is that tumour cells are exfoliated from the primary tumour and seed the intraluminal local resection environment [17]. When perineal sepsis occurs, these cells may find an adequate environment for implementation and growth that leads to pelvic recurrence. In vitro studies have demonstrated a multifactorial mechanism by which inflammatory responses of the postoperative peritoneum and/or pelvic sepsis may enhance local recurrence, and this mechanism involves a combination of the amplification of angiogenesis and stimulation of both the migration and invasion capacities of tumour cells [18]. This is a plausible theory in cases of CPR because no viable tumours are found in the resection specimens, leading us to believe that unique viable tumours are left in the perirectal (or extra-fascial) environment and may be reactivated by postoperative local inflammation.

Poor tumour differentiation is one of the best-known predictive factors of local and distant recurrence [18]. It is also a predictive factor of an incomplete response to neoadjuvant chemoradiotherapy [19]. However, it remains unclear how poorly differentiated tumours (especially the SRCC subtype) enable aggressive outcomes even after a CPR [20, 21]. Linosilva et al. reported a case with the presence of microscopic clinical carcinomatosis implants with a total mural tumour response [21]. This finding is a reminder of the strong potential for SRCCs in other locations and the high probability of recurrence, especially for peritoneal carcinomatosis [22, 23].

Another theory of local and distant recurrence after a CPR may be that the tumour is left behind in the patient during surgery [4]. Lateral pelvic lymph node (LPLN) involvement is associated with poorer survival and a high rate of locoregional recurrence [24-26]. The management of this disease is completely different in Japan than in all other countries. In Western countries, LPLNs are considered metastatic and can only be managed by preoperative chemoradiotherapy combined with a standard TME, whereas in Japan, lateral pelvic lymph node 
dissection (LPLND) represents a standard regional lymphadenectomy. Akiyoshi et al. reported that preoperative CRT alone cannot eradicate LPLN involvement, but excellent local control and survival can be achieved with the combination of preoperative CRT and LPLND [27]. Because MRI before CRT seems to be useful for predicting LPLN metastases, extended lymphadenectomy may be indicated for selected patients with advanced low rectal cancer with preoperative $\mathrm{cN}$ status on a preoperative MRI [28] to avoid recurrence, even after a CPR.

Some limitations should be considered when interpreting our study results, including the retrospective multicenter study design, limited follow-up period, small size of the studied population and potential bias inherent in the data collection and analysis. We acknowledge that deficiencies in the perioperative evaluations may exist, such as the lack of systematic post-CRT MRI examinations, differences in radiotherapy protocols between centres, and difficulties in the pathological analysis of the specimens in cases in which no tumour was found. However, very specific attention was given to all specimens with a $\mathrm{pCR}$, and they were reviewed by a second pathologist according to a standardized protocol.

To our knowledge, this is the first study to suggest that even in the group with the best prognoses and CPR after chemoradiotherapy for low rectal cancer, the occurrence of perineal sepsis and/or poor initial differentiation may be associated with impaired DFS.

Based on these findings, other options for the management of advanced low rectal cancer may be discussed, such as LPLND in patients with pre-treatment cN IPLN status on MRI results and the addition of oxaliplatin to fluorouracil-based neoadjuvant chemoradiotherapy and adjuvant chemotherapy to reduce the risks of both local and distant recurrences [29].

\section{Conclusion}

This study demonstrated that a CPR is associated with a $12.6 \%$ rate of recurrence and that perineal sepsis and histologically poor differentiation are associated with worse DFS times among patients with a CPR. Additional aggressive therapeutic options should be considered when these risk factors are present.

\footnotetext{
Abbreviations

APR: Abdominoperineal resection; ASA score: American Society of Anesthesiologist; CD score: Clavien Dindo score; Cl: Confidence interval; CN: Clinical Node; CPR: Complete pathological response; CRT: Chemoradiotherapy; DFS: Disease-free survival; ESMO: European Society of Medical Oncology; HR: Hazard Ratio; LPLN: Lateral pelvic lymph node; MRI: Magnetic Resonance Imaging; NCCN: National Comprehensive Cancer Network; OR: Ods ratio; SD: Standard Deviation; SRCC: Signet Ring Cancer Cell; TME: Total Mesorectal Excision; UICC: Union Internationale Contre le Cancer
}

\section{Acknowledgements}

Authors would like to thank Misses Hanane Benkhouya, Kawtar Belkhadir and Yasmina Bensouda for their supports.

\section{Authors' contributions}

AmS, MAM, AmB, OHEM, MR, LI, KM, AZ, RM, MR, AbS, AbB, MohA and AH designed the research; AmS, MAM, AmB, BS, TS, BEK, TEH, SMB, MouA performed the research; AmS, MAM, AmB, MouA analyzed the data; AmS and MAM wrote the paper and all the authors have read and approved the manuscript.

\section{Funding}

The authors declare that they had no funding.

\section{Availability of data and materials}

The datasets used and/or analysed during the current study are available from the corresponding author on reasonable request.

\section{Ethics approval and consent to participate}

This study was reviewed and approved by the Ethics Committee of the Ibn Sina Hospital (Rabat, Morocco). The Batna Anti Cancer center (Batna, Algeria) institutional review board waived the ethical approval for this study.

\section{Consent for publication}

The consent to participate is not applicable since this is a retrospective series containing no individual data.

\section{Competing interests}

The authors declare that they have no competing interests.

\section{Author details}

'Surgical Oncology Department, National Institute of Oncology, Mohammed V University Medical School, Rabat, Morocco. ${ }^{2}$ Surgical Oncology, Hospital El Farabi, Mohammed Ist University, Oujda, Morocco. ${ }^{3}$ Surgery Department, Sidi Mohammed Ben Abdellah University, Fes, Morocco. ${ }^{4}$ Surgery Department, Military Hospital, Rabat, Morocco. ${ }^{5}$ Batna Anticancer Center Alger, Batna, Algeria. ${ }^{6}$ Anatomopathology Department, National Institute of Oncology, Rabat, Morocco. "Surgical Department "A", Ibn Sina Hospital, Rabat, Morocco. ${ }^{8}$ Private surgical oncology center, Salé, Morocco. ' ${ }^{9}$ Surgical Department " $C$ ", Ibn Sina Hospital, Mohammed V University. Medical School, Rabat, Morocco.

Received: 20 September 2018 Accepted: 3 October 2019

Published online: 28 October 2019

\section{References}

1. Sauer R, Becker H, Hohenberger W, Rodel C, Wittekind C, Fietkau R, et al. Preoperative versus postoperative chemoradiotherapy for rectal cancer. $\mathrm{N}$ Engl J Med. 2004;351(17):1731-40.

2. Habr-Gama A, Perez RO, Nadalin W, Sabbaga J, Ribeiro U Jr, Silva e Sousa $\mathrm{AH} \mathrm{Jr}$, et al. Operative versus nonoperative treatment for stage 0 distal rectal cancer following chemoradiation therapy: long-term results. Ann Surg. 2004;240(4):711-7 discussion 717-718.

3. Li J, Liu H, Yin J, Liu S, Hu J, Du F, et al. Wait-and-see or radical surgery for rectal cancer patients with a clinical complete response after neoadjuvant chemoradiotherapy: a cohort study. Oncotarget. 2015;6(39):42354-61.

4. Maas M, Nelemans PJ, Valentini V, Das P, Rodel C, Kuo LJ, et al. Long-term outcome in patients with a pathological complete response after chemoradiation for rectal cancer: a pooled analysis of individual patient data. Lancet Oncol. 2010;11(9):835-44.

5. Armstrong D, Raissouni S, Price Hiller J, Mercer J, Powell E, MacLean A, et al. Predictors of pathologic complete response after Neoadjuvant treatment for rectal cancer: a multicenter study. Clin Colorectal Cancer. 2015;14(4):291-5.

6. Martin ST, Heneghan HM, Winter DC. Systematic review and meta-analysis of outcomes following pathological complete response to neoadjuvant chemoradiotherapy for rectal cancer. Br J Surg. 2012;99(7):918-28.

7. Capirci C, Valentini V, Cionini L, De Paoli A, Rodel C, Glynne-Jones R, et al. Prognostic value of pathologic complete response after neoadjuvant therapy in locally advanced rectal cancer: long-term analysis of $566 \mathrm{ypCR}$ patients. Int J Radiat Oncol Biol Phys. 2008;72(1):99-107.

8. Cotte E, Passot G, Decullier E, Maurice C, Glehen O, Francois Y, et al. Pathologic response, when increased by longer interval, is a marker but not 
the cause of good prognosis in rectal cancer: 17-year follow-up of the Lyon R90-01 randomized trial. Int J Radiat Oncol Biol Phys. 2016;94(3):544-53.

9. Agha RA, Fowler AJ, Rajmohan S, Barai I, Orgill DP, Group P. Preferred reporting of case series in surgery; the PROCESS guidelines. Int I Surg. 2016; 36(Pt A):319-23.

10. Souadka A, Majbar MA, El Harroudi T, Benkabbou A, Souadka A. Perineal pseudocontinent colostomy is safe and efficient technique for perineal reconstruction after abdominoperineal resection for rectal adenocarcinoma. BMC Surg. 2015;15:40.

11. Dindo D, Demartines N, Clavien PA. Classification of surgical complications: a new proposal with evaluation in a cohort of 6336 patients and results of a survey. Ann Surg. 2004;240(2):205-13.

12. Paun BC, Cassie S, MacLean AR, Dixon E, Buie WD. Postoperative complications following surgery for rectal cancer. Ann Surg. 2010;251(5): 807-18

13. Pucciarelli S, Toppan P, Friso ML, Russo V, Pasetto L, Urso E, et al. Complete pathologic response following preoperative chemoradiation therapy for middle to lower rectal cancer is not a prognostic factor for a better outcome. Dis Colon Rectum. 2004;47(11):1798-807.

14. Shivnani AT, Small W Jr, Stryker SJ, Kiel KD, Lim S, Halverson AL, et al. Preoperative chemoradiation for rectal cancer: results of multimodality management and analysis of prognostic factors. Am J Surg. 2007;193(3): 389-93 discussion 393-384

15. Lin JK, Yueh TC, Chang SC, Lin CC, Lan YT, Wang HS, et al. The influence of fecal diversion and anastomotic leakage on survival after resection of rectal cancer. J Gastrointest Surg. 2011;15(12):2251-61.

16. Wang $\mathrm{S}$, Liu J, Wang $\mathrm{S}$, Zhao H, Ge S, Wang W. Adverse effects of anastomotic leakage on local recurrence and survival after curative anterior resection for rectal cancer: a systematic review and meta-analysis. World J Surg. 2017;41(1):277-84.

17. Kouraklis G, Glinavou A, Kouvaraki M, Raftopoulos J, Karatzas G. Anal lesion resulting from implantation of viable tumour cells in a pre-existing anal fistula. A case report. Acta Chir Belg. 2002;102(3):212-3.

18. Salvans S, Mayol X, Alonso S, Messeguer R, Pascual M, Mojal S, et al. Postoperative peritoneal infection enhances migration and invasion capacities of tumor cells in vitro: an insight into the association between anastomotic leak and recurrence after surgery for colorectal cancer. Ann Surg. 2014;260(5):939-43 discussion 943-934.

19. Reggiani Bonetti L, Lionti S, Domati F, Pagliani G, Mattioli E, Barresi V. Histological grading based on poorly differentiated clusters is predictive of tumour response and clinical outcome in rectal carcinoma treated with neoadjuvant chemoradiotherapy. Histopathology. 2017;71(3):393-405. https://doi.org/10.1111/his.13242

20. Souadka A, Majbar MA, Raiss M. Can we rely on the adequate mesorectum excision and the complete pathological response in case of rectal signetring cell carcinoma? J Surg Oncol. 2016;114(5):649.

21. Lino-Silva LS, Garcia-Gomez MA, Salcedo-Hernandez RA. In response: can we rely on the adequate mesorectum excision and the complete pathological response in case of rectal signet-ring cell carcinoma. J Surg Oncol. 2016; 114(5):650.

22. Messager M, Lefevre JH, Pichot-Delahaye V, Souadka A, Piessen G, Mariette $C$, et al. The impact of perioperative chemotherapy on survival in patients with gastric signet ring cell adenocarcinoma: a multicenter comparative study. Ann Surg. 2011;254(5):684-93 discussion 693.

23. Honore C, Goere D, Messager M, Souadka A, Dumont F, Piessen G, et al. Risk factors of peritoneal recurrence in eso-gastric signet ring cell adenocarcinoma: results of a multicentre retrospective study. Eur J Surg Oncol. 2013;39(3):235-41.

24. Kim TH, Jeong SY, Choi DH, Kim DY, Jung KH, Moon SH, et al. Lateral lymph node metastasis is a major cause of locoregional recurrence in rectal cancer treated with preoperative chemoradiotherapy and curative resection. Ann Surg Oncol. 2008;15(3):729-37.

25. Akiyoshi T, Watanabe T, Miyata S, Kotake K, Muto T, Sugihara K, et al. Results of a Japanese nationwide multi-institutional study on lateral pelvic lymph node metastasis in low rectal cancer: is it regional or distant disease? Ann Surg. 2012;255(6):1129-34.

26. Yokoyama S, Takifuji K, Hotta T, Matsuda K, Watanabe T, Mitani Y, et al. Survival benefit of lateral lymph node dissection according to the region of involvement and the number of lateral lymph nodes involved. Surg Today. 2014:44(6):1097-103.
27. Akiyoshi T, Ueno M, Matsueda K, Konishi T, Fujimoto Y, Nagayama S, et al. Selective lateral pelvic lymph node dissection in patients with advanced low rectal cancer treated with preoperative chemoradiotherapy based on pretreatment imaging. Ann Surg Oncol. 2014;21(1):189-96.

28. Akiyoshi T, Matsueda K, Hiratsuka M, Unno T, Nagata J, Nagasaki T, et al. Indications for lateral pelvic lymph node dissection based on magnetic resonance imaging before and after preoperative Chemoradiotherapy in patients with advanced low-rectal cancer. Ann Surg Oncol. 2015;22(Suppl 3): S614-20.

29. Rodel C, Graeven U, Fietkau R, Hohenberger W, Hothorn T, Arnold D, et al. Oxaliplatin added to fluorouracil-based preoperative chemoradiotherapy and postoperative chemotherapy of locally advanced rectal cancer (the German CAO/ARO/AlO-04 study): final results of the multicentre, open-label, randomised, phase 3 trial. Lancet Oncol. 2015;16(8):979-89.

\section{Publisher's Note}

Springer Nature remains neutral with regard to jurisdictional claims in published maps and institutional affiliations.
Ready to submit your research? Choose BMC and benefit from:

- fast, convenient online submission

- thorough peer review by experienced researchers in your field

- rapid publication on acceptance

- support for research data, including large and complex data types

- gold Open Access which fosters wider collaboration and increased citations

- maximum visibility for your research: over $100 \mathrm{M}$ website views per year

At $\mathrm{BMC}$, research is always in progress.

Learn more biomedcentral.com/submissions 